\title{
EVALUATION OF RENOPROTECTIVE EFFECT OF CILNIDIPINE IN PATIENTS WITH MILD TO MODERATE HYPERTENSION AND TYPE 2 DIABETES MELLITUS - A PROSPECTIVE STUDY
}

\author{
RAMYA R ${ }^{1}$, SHAJAHAN OM ${ }^{2 *}$, ANAKHA KALADHARAN ${ }^{3}$
}

${ }^{1}$ Department of Pharmacology, Vinayaka Missions Research Foundation, Salem, Tamil Nadu, India. ${ }^{2}$ Clinical Pharmacologist and Basic Science Faculty for DNB, Vijaya Group of Hospitals, Chennai, Tamil Nadu, India. ${ }^{3}$ Pharm D Intern, K. K. College of Pharmacy, Chennai, Tamil Nadu, India. Email: doc.shajahan.om@gmail.com

Received: 09 October 2020, Revised and Accepted: 26 November 2020

ABSTRACT

Objective: The objective of the study was to evaluate the renoprotective effect of cilnidipine by estimating urinary albumin and creatinine levels in mild-to-moderate hypertension (HTN) with type 2 diabetes mellitus (DM) and also evaluate the adverse drug profile of cilnidipine in the same patients.

Methods: This was a single-center, prospective, open-labeled, randomized study. A total of 60 patients of either gender aged between 30 and 60 with mild-to-moderate HTN with type 2 DM were included in the study. Urine albumin and urine creatinine were measured at day 1 and day 181 . Blood pressure (BP) was measured in all visits. The drug cilnidipine at a dose of 10-20 mg oral was given and the corresponding improvement in the levels of urine albumin and other parameters was identified.

Results: There was a significant reduction in the mean systolic BP from $150.07 \pm 5.44 \mathrm{mmHg}$ in visit 0 to $123.03 \pm 5.23 \mathrm{mmHg}$ in visit 3 . And also, there was a significant reduction in the mean diastolic BP from $95.5 \pm 8.15 \mathrm{mmHg}$ in visit 0 to $80.8 \pm 2.42 \mathrm{mmHg}$ in visit 3 . The mean heart rate at visit zero was $76.71 \pm 4.86$. At the end of 6 months of treatment, there was a significant reduction to $70.63 \pm 2.74$. There was a significant reduction in the microalbuminuria from $66.62 \pm 8.39$ to $38.8 \pm 6.45$. The mean reduction was $27.56 \pm 10.25$. There was no change in the creatinine level.

Conclusion: The study reveals that the drug cilnidipine is safe and effective in reducing the microalbuminuria and also effectively reduces BP in hypertensive patients. Hence, the drug cilnidipine can be safely administered to the patient with diabetes and HTN

Keywords: Cilnidipine, Renoprotective, Microalbuminuria, Hypertension, Diabetes mellitus.

(C) 2021 The Authors. Published by Innovare Academic Sciences Pvt Ltd. This is an open access article under the CC BY license (http://creativecommons.org/ licenses/by/4.0/) DOI: http://dx.doi.org/10.22159/ajpcr.2021v14i1.39962. Journal homepage: https://innovareacademics.in/journals/index.php/ajpcr

\section{INTRODUCTION}

Hypertension (HTN) and diabetes mellitus (DM) are a worldwide major public health problem and a major risk factor for renal failure and other organ damage. In a prospective cohort study, the development of type II diabetes was almost 2.5 times as likely in persons with HTN than in normotensive patients [1]. This is in coincidence with substantial evidence of the increased prevalence of HTN in diabetic persons [2]. The risk for cardiovascular disease (CVD) is quadruplicate higher in patients with both DM and HTN as compared to the normotensive nondiabetic controls $[3,4]$. Diabetic nephropathy and microalbuminuria are found to be strong predictors and vital indicators of cardiovascular and also overall morbidity and mortality in patients of diabetes. Angiotensin-converting enzyme (ACE) inhibitors and angiotensin II receptor blockers (ARBs) can be used in case of albuminuria. Although ACE inhibitors and ARBs reduce proteinuria significantly and equally effective in lowering blood pressure (BP), many adverse effects are noticed with these drugs. The use of ACE inhibitors or ARBs may exacerbate hyperkalemia in patients and are contraindicate in advanced renal insufficiency. ACE inhibitors may cause a rapid decline in renal function, hence, they are contraindicated in older patients with bilateral renal artery stenosis. ARBs can cause hyperkalemia in patients with renal failure and in patients taking potassium-sparing diuretics. When the patients do not respond to ARB monotherapy, diuretics or non-dihydropyridine calcium channel blockers (CCBs) are the second line of drugs. However, diuretics can cause metabolic side effects and non-dihydropyridine CCBs can cause a negative ionotropic effect. The dihydropyridine CCB, amlodipine is a widely used drug but has no renoprotective property. Cilnidipine is a novel and unique dihydropyridine derivative CCB blocker that blocks both
L-type and N-type voltage-dependent calcium channels [5]. It inhibits sympathomimetic activity in contrast to other dihydropyridines. And also, the incidence of ankle edema is low with cilnidipine when compared to amlodipine [6].

The present study was, therefore, designed and conducted to evaluate the renoprotective effect of cilnidipine by estimating urinary albumin and serum creatinine levels in hypertensive patients with concomitant type $2 \mathrm{DM}$ and albuminuria.

\section{METHODS}

This was a prospective, open-labeled, randomized study conducted for 1 year in Vinayaka Missions Kirupananda Variyar Medical College and Hospital, Salem. A total of 60 patients of either gender aged between 30 and 60 with mild-to-moderate HTN and type 2 DM were included in the study.

\section{Inclusion Criteria}

Mild-to-moderate HTN patients with type 2 DM (either gender aged between 30 and 60 years) and microalbuminuria in the range of 30$300 \mathrm{mg} / \mathrm{L}$ are included in the study.

\section{Exclusion Criteria}

Hypertensive subjects with systolic BP (SBP) $\geq 180 \mathrm{mmHg}$ and/ or diastolic BP (DBP) $\geq 110 \mathrm{mmHg}$, subjects on two or more antihypertensive medications, pregnant and lactating women, those with severe heart failure or with severe liver dysfunction, those with end-stage renal disease, and those undergoing dialysis were excluded from the study. 
After a detailed history, physical examination, clinical examination (SBP, DBP, and heart rate), and laboratory investigation (Hb, TC, DC, ESR, LFT, RFT, and ECG), the recruited patients were given cilnidipine 10-20 mg once daily $1 / 2 \mathrm{~h}$ after breakfast for 6 months. Patients enrolled in the study were not permitted to use any other medications apart from the antihypertensive drugs given to them. All the recruited patients completed the study.

The study protocol was approved by the Institutional Ethics Committee VMKVMC/IEC/14/8. Informed consent was obtained from the patient before conducting the study by explaining the study procedures to the patient.

\section{Patient Monitoring}

$\mathrm{BP}$, heart rate, proteinuria, and serum creatine were measured in all patients. There were four visits (Visit 0 at the start of the study, Visit 1 at 1 month, visit 2 at 3 months and Visit 3 at 6 months). During each visit, the SBP, DBP, and heart rate were measured. Urine albumin and serum creatinine were measured at day 1 and 6 months. Microalbuminuria can be diagnosed from elevated concentrations in a spot sample (30$299 \mathrm{mg} / \mathrm{L}$ ). Urine albumin levels were estimated by latex turbidimetrymicroalbumin-turbilatex test (Euro Diagnostics). The drug cilnidipine at a dose of 10-20 mg oral was given to all patients and the corresponding improvement in the levels of urine albumin and other parameters were identified.

\section{Statistical Analysis}

All parameters such as age, body mass index (BMI), BP, heart rate, proteinuria, fasting blood sugar, postprandial blood sugar, and serum creatinine were expressed as mean \pm standard deviation (SD).

\section{RESULTS}

\section{Demographic Data}

In about 60 patients, there were 38 (63.33\%) males and 22 (36.6\%) were females. Patients between the age of 45 and 55 were selected. Mean age of the patient recruited for the study was $49.36 \pm 6.08$. About $13 \%$ of the patients had the habit of smoking. These baseline demographic data are shown in Table 1.

Table 1: Baseline demographic and clinical characteristics of patients

\begin{tabular}{ll}
\hline Baseline demographic data & Mean \pm SD \\
\hline Age (years) & $49.36 \pm 6.089$ \\
Sex & $38(63.33 \%)$ males \\
& $22(36.6 \%)$ females \\
Weight $(\mathrm{Kg})$ & $65.1 \pm 10.11$ \\
Height $(\mathrm{cm})$ & $162.07 \pm 6.37$ \\
BMI $\left(\mathrm{Kg} / \mathrm{m}^{2}\right)$ & $24.77 \pm 3.44$ \\
Heart rate (beats/min) & $76.71 \pm 4.86$ \\
Smokers (\%) & $8(13 \%)$ \\
Fasting blood sugar (mg/dl) & $140.13 \pm 9.24$ \\
Postprandial blood sugar (mg/dl) & $227.88 \pm 17.28$ \\
SBP mmHg & $150.07 \pm 5.44$ \\
DBP mmHg & $95.5 \pm 8.15$ \\
Albuminuria (mg/L) & $66.62 \pm 8.39$ \\
HbA1C (\%) & $8.05 \pm 0.87$ \\
Creatinine (mg/dl) & $0.88 \pm 0.11$ \\
\hline
\end{tabular}

SD: Standard deviation; BMI: Body mass index; SBP: Systolic blood pressure; DBP: Diastolic blood pressure

\section{Efficacy Evaluation}

Table 2 shows that there was a significant reduction in the mean SBP from $150.07 \pm 5.44 \mathrm{mmHg}$ in visit 0 to $123.03 \pm 5.23 \mathrm{mmHg}$ in visit 3 (after 6 months of treatment). And also, there was a significant reduction in the mean DBP from $5.5 \pm 8.15 \mathrm{mmHg}$ in visit 0 to $80.8 \pm 2.42 \mathrm{mmHg}$ in visit 3 (after 6 months of treatment). There was a significant reduction in both systolic and DBP in the first, second, and third visits. The mean heart rate at visit zero was $76.71 \pm 4.86$. At the end of 6 months of treatment, there was a significant reduction to $70.63 \pm 2.74$. There was a significant reduction in the microalbuminuria from $66.62 \pm 8.39$ to $38.8 \pm 6.45$. The mean reduction was $27.56 \pm 10.25$. There was no change in the creatinine level.

\section{Safety Evaluation}

Table 3 demonstrates the adverse effects suffered by patients with cilnidipine. The common adverse effects seen with cilnidipine were headache, nausea, and fatigue. None of the patients had ankle edema.

\section{DISCUSSION}

Cilnidipine was extensively studied by researchers in its preclinical and clinical developmental stages and is still being studied. It was proved to have a reno, neuro, and cardioprotective effect - decrease in heart rate, apart from its BP lowering effect. Hence, a study was conducted to evaluate the renoprotective effect of cilnidipine in patients with mild-to-moderate HTN and type 2 DM. This study revealed that microalbuminuria was significantly reduced with cilnidipine administration and there was no serious adverse effect with cilnidipine which was reported. The study also confirmed that the cilnidipine is safe and effective in reducing microalbuminuria in hypertensive patients.

A study by Xu et al. [7] has shown that cilnidipine was well tolerated by hypertensive patients with a very minimal adverse event such as headache, dizziness, cough, and gastrointestinal disturbances which are comparable to amlodipine [8]. Hence, CCBs with action on N-type calcium channel can cause dilation of venules through the sympathetic system and can reduce the incidence of pedal edema compared to CCBs that act only on L-type calcium channels. Even in our study, the patients had minimal adverse effects and no patients experienced pedal edema.

Kojima et al. [8] and Tsuchihashi et al. [9] studied the renoprotective effect of cilnidipine and found outstanding results. They reported that cilnidipine had a better renoprotective effect when compared to pure L-type CCBs. It reduces glomerular filtration pressure, suppresses renin-angiotensin-aldosterone secretion, and reduces the incidence of proteinuria.

In the study by Tanaka [10], cilnidipine had a renoprotective effect compared with other $\mathrm{CCB}$ and concluded that heart rate and albuminuria were decreased following a switch from other CCB to cilnidipine without any change in BP. In the present study, there is a significant reduction in albuminuria with cilnidipine. However, the present study was not a comparative study.

Arijit et al. [11] compared the effectiveness of amlodipine and cilnidipine in patients with essential HTN and their effect on heart rate and serum uric acid levels. The study results showed that the patient had a significant reduction in heart rate and serum uric acid levels from baseline. Sarkar et al. [11] compared the effect of CCBs amlodipine

Table 2: Effect of cilnidipine on BP, heart rate, albuminuria, and serum creatinine

\begin{tabular}{lllll}
\hline Parameters & Visit 0 & Visit1 & Visit 2 & Visit 3 \\
\hline SBP (mmHg) & $150.07 \pm 5.44$ & $138.47 \pm 6.86$ & $128.9 \pm 5.95$ & $123.03 \pm 5.23$ \\
DBP (mmHg) & $95.5 \pm 8.15$ & $87.77 \pm 4.73$ & $83.27 \pm 3.90$ & $80.8 \pm 2.42$ \\
Heart rate (Beats per min) & $76.71 \pm 4.86$ & $75 \pm 3.98$ & $73.1 \pm 3.176$ & $70.63 \pm 2.74$ \\
Albuminuria (mg/L) & $66.62 \pm 8.39$ & - & - & $38.8 \pm 6.45$ \\
Creatinine (mg/dl) & $0.88 \pm 0.11$ & - & - & $0.89 \pm 0.11$ \\
\hline
\end{tabular}

BP: Blood pressure; SBP: Systolic blood pressure; DBP: Diastolic blood pressure 
Table 3: Data related to adverse effects

\begin{tabular}{ll}
\hline Adverse effect & No (\%) \\
\hline No ADR & 40 \\
Headache & 6 \\
Fatigue & 4 \\
Nausea & 3 \\
Vomiting & 1 \\
Abdominal pain & 2 \\
Fatigue+nausea & 3 \\
Headache+nausea & 1 \\
Total & 60 \\
\hline
\end{tabular}

ADR: Adverse drug reaction

and cilnidipine and found that the elevation of creatinine was more suppressed by the cilnidipine than amlodipine. The study concluded that cilnidipine has better renoprotective profile than amlodipine. This is comparable with our study that showed no change in creatinine level.

Soeki et al. [12] compared the antioxidant and antiproteinuric effects of cilnidipine and amlodipine in hypertensive patients. The study suggested that cilnidipine has a greater antiproteinuric effect than the amlodipine when used in combination with renin-angiotensin system inhibitor.

In a study conducted by Singh et al. [13], there was a greater reduction in microalbuminuria by both enalapril and cilnidipine together than enalapril alone. Our study, Shajahan et al. [14] showed that there was a significant reduction in heart rate for patients treated with cilnidipine. Furthermore, there was a significant reduction in both systolic and DBP. Adverse events were less, and tolerability was better with cilnidipine.

Albuminuria is an independent risk for cardiovascular events. It was already reported that an increase in albuminuria elevates the risk of developing CVD and death. Reducing albuminuria through treatment reduces the risk. Even with highest normal level of albuminuria, the risk for cardiovascular events is increased. From the study, it is well understood that cilnidipine can reduce BP, elevated creatinine levels, heart rate, and microalbuminuria. Hence, cilnidipine is considered to have a renoprotective effect.

\section{Limitations of the Study}

The main drawback of our study was that it was not a comparative study. The other limitations are single-centered, open-labeled study with a small number of patients. Hence, an attempt should be made to extend the study with a large number of patients in multicenter with comparative groups.

\section{CONCLUSION}

From this study, we can conclude that cilnidipine can significantly reduce $\mathrm{BP}$ and microalbuminuria in mild-to-moderate hypertensive patients with DM. Hence, it can be used in renal compromised patients.

\section{ACKNOWLEDGMENT}

We would like to show our gratitude to Dr. Vijay Babu sir, Professor, Annapoorna Medical College and Hospitals, Salem, Tamil Nadu. We are also immensely grateful to him for his support and encouragement.

\section{AUTHORS' CONTRIBUTIONS}

All authors have contributed equally to bring this original article effectively.

\section{CONFLICTS OF INTEREST}

The authors had no conflicts of interest to declare in relation to this article.

\section{AUTHORS' FUNDING}

We did not receive any funding for the present original article.

\section{REFERENCES}

1. The National High Blood Pressure Education Program Working Group. National high blood pressure education program working group report on hypertension in diabetes. Hypertension 1994;23:145-58.

2. UK Prospective Diabetes Study Group. Tight blood pressure control and risk of macrovascular and microvascular complications in type 2 diabetes: UKPDS 38. UK Prospective Diabetes Study Group. BMJ 1998;317:703-13.

3. Stamler J, Vaccaro O, Neaton JD, Wentworth D. Diabetes, other risk factors, and 12-yr cardiovascular mortality for men screened in the multiple risk factor intervention trial. Diabetes Care 1993;16:434-44.

4. Hu G, Jousilahti P, Tuomilehto J. Joint effects of history of hypertension at baseline and Type 2 diabetes at baseline and during follow-up on the risk of coronary heart disease. Eur Heart J 2007;28:3059-66.

5. Minami J, Ishimitsu T, Kawano Y, Numabe A, Matsuoka H. Comparison of 24-hour blood pressure, heart rate, and autonomic nerve activity in hypertensive patients treated with cilnidipine or nifedipine retard. J Cardiovasc Pharmacol 1998;32:331-6.

6. Adake P, Somashekar HS, Mohammed Rafeeq PK, Umar D, Basheer B, Baroud K. Comparison of amlodipine with cilnidipine on antihypertensive efficacy and incidence of pedal edema in mild to moderate hypertensive individuals: A prospective study. J Adv Pharm Technol Res 2015;6:81-5.

7. Xu GL, Hui X, Wu HD, Ling Q. A meta-analysis of the efficacy and safety of cilnidipine in Chinese patients with mild to moderate essential hypertension. Afr J Pharm Pharmacol 2012;6:2393-9.

8. Kojima S, Shida M, Yokoyama H. Comparison between cilnidipine and amlodipine besilate with respect to proteinuria in hypertensive patients with renal diseases. Hypertens Res 2004;27:379-85.

9. Tsuchihashi T, Ueno M, Tominaga M, Kajioka T, Onaka U, Eto K, et al. Anti-proteinuric effect of an N-type calcium channel blocker, cilnidipine. Clin Exp Hypertens 2005;27:583-91.

10. Tanaka $\mathrm{M}$. The $\mathrm{L} / \mathrm{N}$-type calcium channel blocker, cilnidipine, reduces heart rate and albuminuria in patients with Type 2 diabetes. J Int Med Res 2010;38:602-10.

11. Das A, Kumar P, Kumari A, Chandra S, Gari M, Singh N, et al. Effects of cilnidipine on heart rate and uric acid metabolism in patients with essential hypertension. Cardiol Res 2016;7:167-72.

12. Soeki T, Kitani M, Kusunose K, Yagi S, Taketani Y, Koshiba K. Renoprotective and antioxidant effects of cilnidipine in hypertensive patients. Hypertens Res 2012;35:1058-62.

13. Singh VK, Mishra A, Gupta KK, Misra R, Patel ML, Shilpa. Reduction of microalbuminuria in type-2 diabetes mellitus with angiotensinconverting enzyme inhibitor alone and with cilnidipine. Indian $\mathrm{J}$ Nephrol 2015;25:334-8.

14. Shajahan OM, Rajaram S, Ramya R, Ranga Bhashyam SR. A comparative study on efficacy and safety of amlodipine and cilnidipine in the treatment of mild to moderate essential hypertension. Natl J Basic Med Sci 2020;10:112-23. 